\title{
A bibliometric analysis on studies of nanomedicine applications in head and neck squamous cell carcinoma
}

\author{
Xiaolan Gao ${ }^{1 \#}$, Shizhe Wang ${ }^{2 \#}$, Zhuowei Tian ${ }^{3}$, Yunteng Wu ${ }^{3}$, Wei Liu ${ }^{3}$ \\ ${ }^{1}$ Department of Stomatology, Maternal and Child Health Hospital of Hubei Province, Wuhan, China; ${ }^{2}$ Department of Orthodontics, Shanghai \\ Ninth People's Hospital, College of Stomatology, Shanghai Jiao Tong University School of Medicine, Shanghai, China; ${ }^{3}$ Department of Oral \\ and Maxillofacial-Head and Neck Oncology, Shanghai Ninth People's Hospital, College of Stomatology, Shanghai Jiao Tong University School \\ of Medicine, National Clinical Research Center for Oral Diseases, Shanghai Key Laboratory of Stomatology \& Shanghai Research Institute of \\ Stomatology, Shanghai, China \\ Contributions: (I) Conception and design: W Liu, Y Wu; (II) Administrative support: Y Wu, W Liu; (III) Provision of study materials or patients: X \\ Gao, S Wang; (IV) Collection and assembly of data: X Gao, S Wang, Z Tian; (V) Data analysis and interpretation: X Gao, S Wang; (VI) Manuscript \\ writing: All authors; (VII) Final approval of manuscript: All authors. \\ "These authors contributed equally to this work. \\ Correspondence to: Dr. Wei Liu; Dr. Yunteng Wu. Department of Oral and Maxillofacial-Head and Neck Oncology, Shanghai Ninth People's Hospital, \\ 639 Zhizaoju Road, Shanghai 200011, China. Email: liuweb@hotmail.com; wuyunteng731@163.com.
}

Background: Bibliometric analysis highlights the pivotal studies and topics that have shaped the understanding and management of a disease in its designated field. Herein, original articles on the applications of nanotechnology in head and neck squamous cell carcinoma (HNSCC) were characterized and analyzed.

Methods: A comprehensive search was carried out using a bibliometric methodology, and eligible articles on nanomedicine research in HNSCC were retrieved from the Scopus database.

Results: A total of 309 eligible articles were retrieved, and the total citation count was 7,468. An ascending trend in citation count was observed since 2004, which increased substantially between 2014 and 2019. There were $144(52.7 \%)$ original articles on oral cavity carcinomas. Chemotherapy ( $\mathrm{n}=53,19.4 \%)$, chemoprevention $(n=35,11.3 \%)$, and photodynamic therapy $(n=23,8.4 \%)$ were the three most published topics on the applications of nanotechnology in the therapy of HNSCC. Sentinel node detection ( $\mathrm{n}=25,9.2 \%)$ and noninvasive cancer diagnosis $(\mathrm{n}=22,8.1 \%)$ were the two most published topics in the diagnostic area of HNSCC. A vast majority of these articles were preclinical studies, and only four articles (1.5\%) were phase I/II clinical studies on chemotherapy and radiotherapy.

Conclusions: To the best of our knowledge, this is the first study to analyze the bibliometric characteristics of original articles on nanomedicine in HNSCC. The results of this study not only provide a historical perspective on the scientific evolution in this field, but also revealed the trends and key topics within it that may help facilitate further research.

Keywords: Bibliometric analysis; head and neck squamous cell carcinoma (HNSCC); nanomedicine; nanotechnology; oral cancer

Submitted Sep 07, 2020. Accepted for publication Nov 16, 2020.

doi: $10.21037 /$ tcr-20-2852

View this article at: http://dx.doi.org/10.21037/tcr-20-2852 


\section{Introduction}

Head and neck squamous cell carcinoma (HNSCC) remains a major cause of cancer morbidity and mortality worldwide (1). Carcinomas located in the oral cavity/mouth, lips, oropharynx, hypopharynx, and larynx, account for over $90 \%$ of head and neck malignancies (2). The global 5 -year survival rate of HNSCC remains approximately $50-60 \%$, despite efforts to improve diagnosis and treatment. Conventional treatment strategies involve surgery, radiotherapy, chemotherapy, or a combination of these therapies (3). Currently, the most effective way to reduce mortality from HNSCC is early diagnosis and timely treatment (4). To overcome the limitations in conventional diagnosis and treatment, there is an urgent need for novel effective strategies against this disease $(3,4)$. Nanotechnologybased systems as diagnostic and therapeutic agents present a viable alternative. Indeed, several nanomedicine platforms have offered the potential to improve diagnostic accuracy, therapeutic efficacy, and tolerability (5-10).

Bibliometric analysis utilizes citation data to assess the academic impact of an article in its designated field, and highlights the pivotal studies and topics that have shaped the management and knowledge of a disease of interest $(11,12)$. Researchers and clinicians do not always evaluate the quality of articles, and may encounter difficulty in identifying the key studies in their area of interest (13). Bibliometric analysis enables scholars to explore the latest research and to become familiar with the research hotspots in a given field. It is a distinctly helpful tool that uses the pattern and frequency of citations as objective indicators for assessing academic achievement within an academic area $(14,15)$. In addition, the citation count of papers can effectively determine which are the key journals, authors, contributing institutions, and countries within a specific academic community (16).

An increasingly large number of studies on nanomedicine applications in HNSCC have been published; for example, a bibliometric analysis of nanomedicine research in triplenegative breast cancer (TNBC) was recently conducted between 2012 and 2017 (17). However, no bibliometric analysis has been reported for HNSCC thus far. Therefore, the objective of the present study was to analyze the characteristics of nanotechnology applications in HNSCC using bibliometric methods, and highlight the study topics and trends in this field.

\section{Methods}

\section{Data source}

As per the Teles et al. (17) method used in the bibliometric analysis of TNBC, we utilized the Scopus database to acquire citation information of research articles on nanomedicine applications in HNSCC. We searched the Scopus citation index up to January 1, 2020 with no restriction on publication year using the following search strategy: TITLE-ABS (nano*) AND TITLE (head neck OR oropharyn* OR hypopharyn* OR laryn* OR oral OR mouth OR intraoral OR tongue OR lip OR buccal OR HNSCC OR OSCC) AND ALL (cancer). The articles were categorized in descending order based on their number of citations. Title and abstract or the full text of the articles was screened and reevaluated to verify each article's correlation with nanomedicine research in HNSCC. The full-text articles were retrieved, and the following information was identified: title, authorship, keywords, year of publication, citation count, citation density (citations per year), institution and country of origin, and journal of publication and its impact factor (IF, Journal of Citation Reports).

\section{Data extraction}

Two independent authors (XG and SW) performed the screening, and subsequently analyzed the information of the articles. In case of discrepancies, the opinion of a third author (ZT) was required to attain consensus. To better assess the academic impact of an article in the nanomedicine field, all the relevant articles were classified as primary research studies (preclinical/basic or clinical) or secondary research studies (narrative review, systematic review, or meta-analysis). Articles were included in the analysis if they were primary research studies, and excluded if they were secondary research articles, letters, or conference papers. The current study did not require any animal or human ethics committee approval.

\section{Statistical analysis}

Data collection was conducted using the Microsoft Office Excel package, and descriptive statistics were used to analyze the bibliometric characteristics. 
A No. of articles
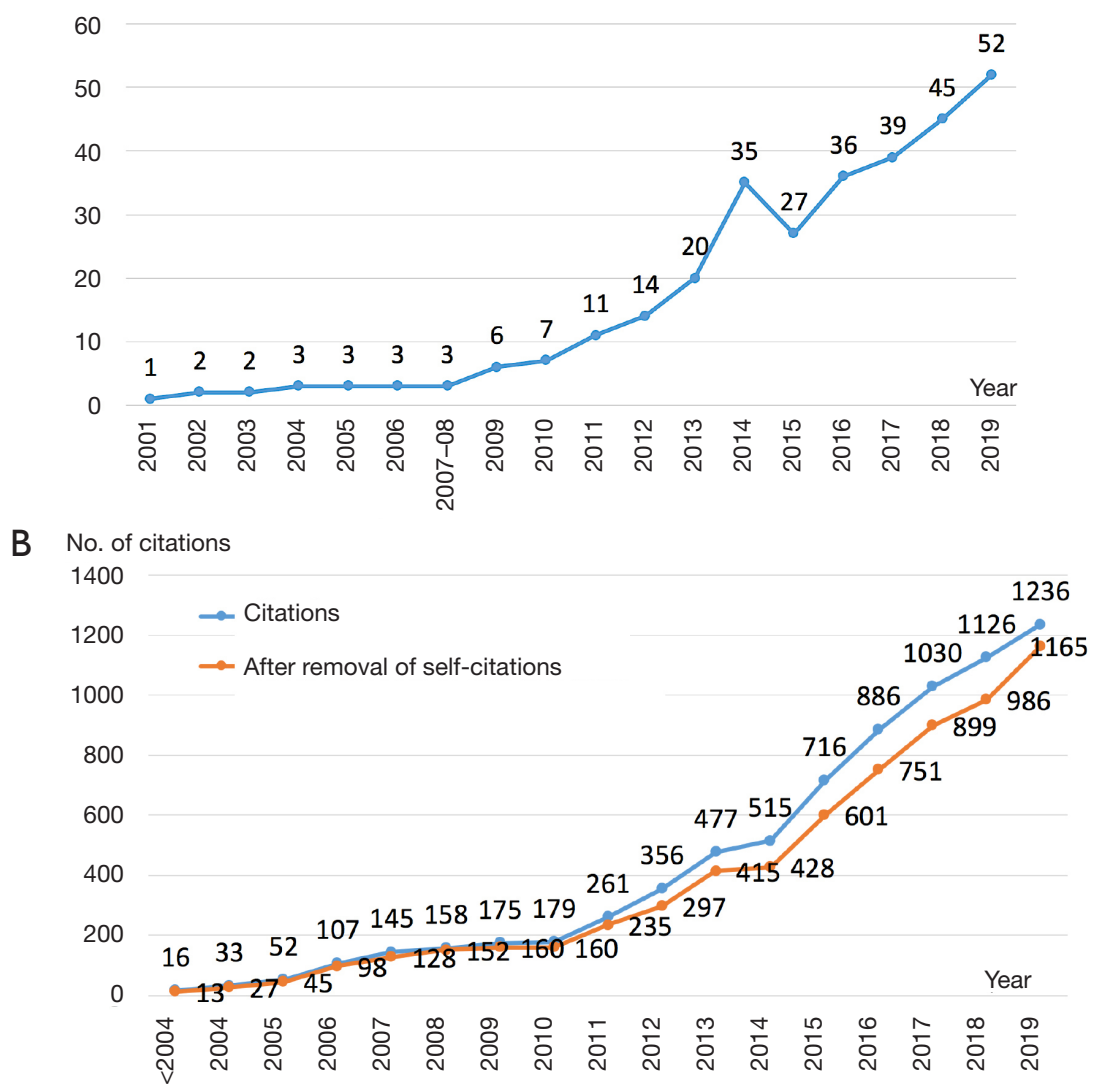

Figure 1 Citation characteristics of original articles on nanomedicine research in head and neck squamous cell carcinoma (HNSCC). (A) The number of papers by year of publication. (B) The total number of citations and the total number of citations after removal of selfcitations by year of publication.

\section{Results}

\section{General citation information}

With the search strategy algorithm, a total of 309 original articles on nanomedicine research in HNSCC were identified from the Scopus citation index between 2001 and 2019. The number of eligible original articles by year of publication is shown in Figure 1A. The majority (72.5\%) of the articles were published in the past 5 years [2014-2019]. There were 38 articles that had not been cited, and 235 articles that had been cited. The total citation count and the total citation count after removal of self-citations were 7,468 and 6,560, respectively. A substantial increase in the number of citations was observed starting from 2004 (Figure 1B). The $b$ index and the $b$ index after removal of self-citations was 39 and 36, respectively. The range of the citation count was 0 to 1,469 (ranking No. 1), with an average number of
28.0. The article with the most citations was a diagnostic study by El-Sayed et al. published in 2005 in the journal, Nano Letters (18), which investigated surface plasmon resonance scattering and absorption of anti-epidermal growth factor receptor (EGFR) antibody-conjugated gold nanoparticles in oral cancer diagnostics. Information on first author, title, publication year, journal and IF, citation count, and citation density of the top-10 cited articles is shown in Table 1, respectively.

\section{Study topic and area}

To analyze study topics and areas of nanomedicine research in HNSCC, the titles and abstracts of each eligible article were screened and evaluated. Chemotherapy ( $\mathrm{n}=53,19.4 \%)$ was the most common topic among eligible articles. The first study to investigate nanotechnology applications 
Table 1 List of top-10 cited original articles on nanomedicine research on HNSCC

\begin{tabular}{|c|c|c|c|c|c|}
\hline First author & Title & $\begin{array}{l}\text { Year of } \\
\text { publication }\end{array}$ & $\begin{array}{l}\text { Journal (abbreviated } \\
\text { name) }\end{array}$ & $\begin{array}{l}\text { Scopus } \\
\text { citations }\end{array}$ & $\begin{array}{l}\text { Citation } \\
\text { density }\end{array}$ \\
\hline El-Sayed I.H., et al. & $\begin{array}{l}\text { Surface plasmon resonance scattering and absorption } \\
\text { of anti-EGFR antibody conjugated gold nanoparticles in } \\
\text { cancer diagnostics: Applications in oral cancer }\end{array}$ & 2005 & Nano Lett & 1,469 & 104.9 \\
\hline Lu K., et al. & $\begin{array}{l}\text { Nanoscale metal-organic framework for highly effective } \\
\text { photodynamic therapy of resistant head and neck } \\
\text { cancer }\end{array}$ & 2014 & $J$ Am Chem Soc & 192 & 38.4 \\
\hline Stoeckli S.J., et al. & $\begin{array}{l}\text { Is there a role for positron emission tomography } \\
\text { with } 18 \mathrm{~F} \text {-fluorodeoxyglucose in the initial staging of } \\
\text { nodal negative oral and oropharyngeal squamous cell } \\
\text { carcinoma }\end{array}$ & 2002 & Head Neck & 145 & 8.5 \\
\hline Wang B., et al. & $\begin{array}{l}\text { Rose-bengal-conjugated gold nanorods for in vivo } \\
\text { photodynamic and photothermal oral cancer therapies }\end{array}$ & 2014 & Biomaterials & 116 & 23.2 \\
\hline Hakim M., et al. & Diagnosis of head-and-neck cancer from exhaled breath & 2011 & Br J Cancer & 105 & 13.1 \\
\hline Damascelli B., et al. & $\begin{array}{l}\text { Intraarterial chemotherapy with polyoxyethylated castor } \\
\text { oil free paclitaxel, incorporated in albumin nanoparticles } \\
\text { (ABI-007): Phase I study of patients with squamous } \\
\text { cell carcinoma of the head and neck and anal canal: } \\
\text { Preliminary evidence of clinical activity }\end{array}$ & 2001 & Cancer & 105 & 5.8 \\
\hline Kah J.C.Y., et al. & $\begin{array}{l}\text { Early diagnosis of oral cancer based on the surface } \\
\text { plasmon resonance of gold nanoparticles }\end{array}$ & 2007 & Int J Nanomed & 102 & 8.5 \\
\hline
\end{tabular}

EGFR, epidermal growth factor receptor; HNSCC, head and neck squamous cell carcinoma.

in the chemotherapy of HNSCC was a phase I study by Damascelli et al. published in 2001 in the journal, Cancer (19). This study reported intra-arterial chemotherapy with polyoxyethylated castor oil-free paclitaxel incorporated in albumin nanoparticles (ABI-007) in patients with squamous cell carcinoma of the head and neck and anal canal. Other topics in the top 10 list included chemoprevention $(\mathrm{n}=35,12.8 \%)$, sentinel node detection $(\mathrm{n}=25,9.2 \%)$, photodynamic therapy $(\mathrm{n}=23,8.4 \%)$, non-invasive cancer diagnosis $(\mathrm{n}=22,8.1 \%)$, photothermal therapy $(\mathrm{n}=16,5.9 \%)$, and radiotherapy $(\mathrm{n}=12,4.4 \%)$ (Figure $2 A)$.

On the basis of the frequency of keywords in the eligible articles, "animal" and "in vitro cell" were the most common keywords, and "oral", "head and neck", "nanoparticles", "drug delivery/carrier", and "antineoplastic agent" were also observed in the top 10 list (Figure 2B). Interestingly, more than half $(\mathrm{n}=144,52.7 \%)$ of all articles were focused on carcinomas located in the oral cavity, while the other articles involved carcinomas of the head and neck, oropharynx, hypopharynx, and larynx. Strikingly, a vast majority of these articles were preclinical/basic studies, and only four articles (1.5\%) were phase I/II clinical studies.

\section{Authorship, contributing institution and country, and journal of publication}

Trends in authorship, contributing institution, and country within a particular scientific community can usually be discerned by bibliometric analysis. The contributing author with largest number of articles was Krishnakumar N. (n=11), followed by Klop WMC. (n=7), Gohulkumar M. $(n=6)$, Gurushankar K. $(n=6)$, and Shin DM. $(n=6)$. The contributing country of origin with the maximum number of articles was China ( $\mathrm{n}=77,28.2 \%)$, followed by 
A

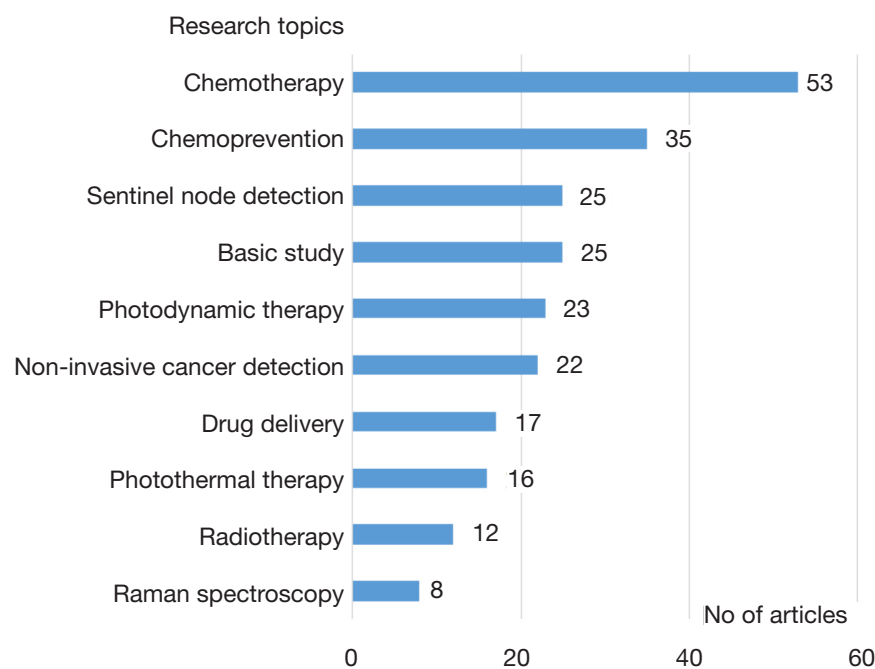

B

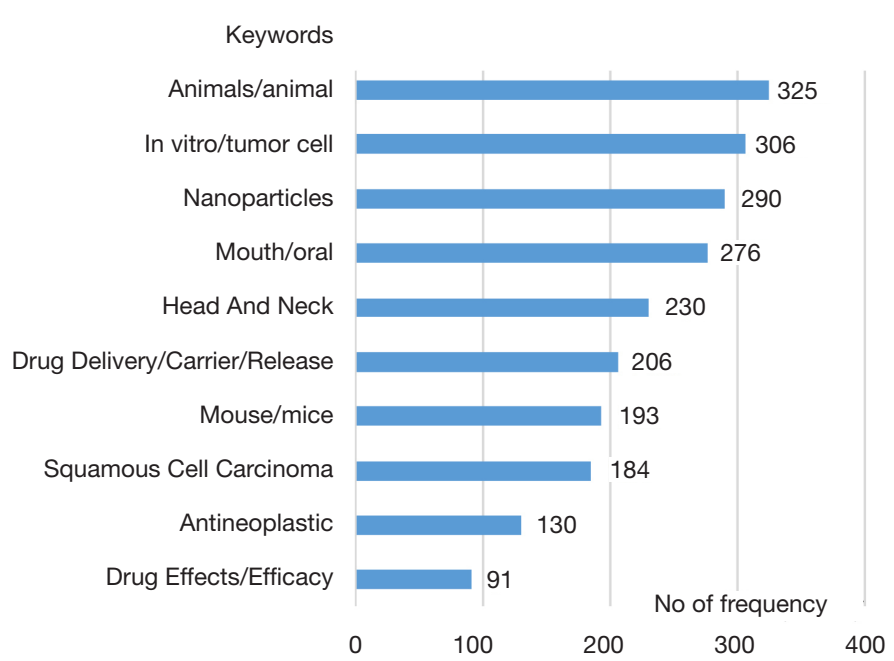

Figure 2 Topics and areas of study of the original articles on nanomedicine research in head and neck squamous cell carcinoma (HNSCC). (A) Top 10 research topics. (B) Top 10 keywords.

the United States ( $\mathrm{n}=69,25.3 \%)$, India $(\mathrm{n}=40,16.7 \%)$, The Netherlands ( $\mathrm{n}=15)$, and Germany $(\mathrm{n}=14)$. The contributing institution of origin with the maximum number of articles was Annamalai University ( $\mathrm{n}=15)$, followed by Jilin University $(\mathrm{n}=10)$, Peking University $(\mathrm{n}=10)$, and Emory University $(\mathrm{n}=8)$. Information on the highest ranked (top 10) authors, countries, and institutions of origin are shown in Figure 3.

Bibliometric analysis often provides a guide for the journal selection of publications. The 309 articles were published across 160 different academic journals. International Fournal of Nanomedicine $(\mathrm{n}=16)$ was the journal of publication with the most number of articles, followed by Nanomedicine $(\mathrm{n}=8)$, Biomaterials $(\mathrm{n}=6)$, and Head and Neck $(\mathrm{n}=6)$. For the journal IF, fournal of the American Chemical Society (IF =14.357) was the journal with the highest IF, followed by ACS Nano (IF =13.709), Advanced Functional Materials ( $\mathrm{IF}=13.325)$, Nature Communications ( $\mathrm{IF}=12.353$ ), and Angewandte Chemie International Edition (IF =12.102). Information on the journals with the highest number of published articles (top 10) and the highest IFs are shown in Figure 4.

\section{Discussion}

Bibliometric analysis is a helpful tool for assessing research trends and for understanding the vital topics in an area of interest that has undergone an academic evolution 
A

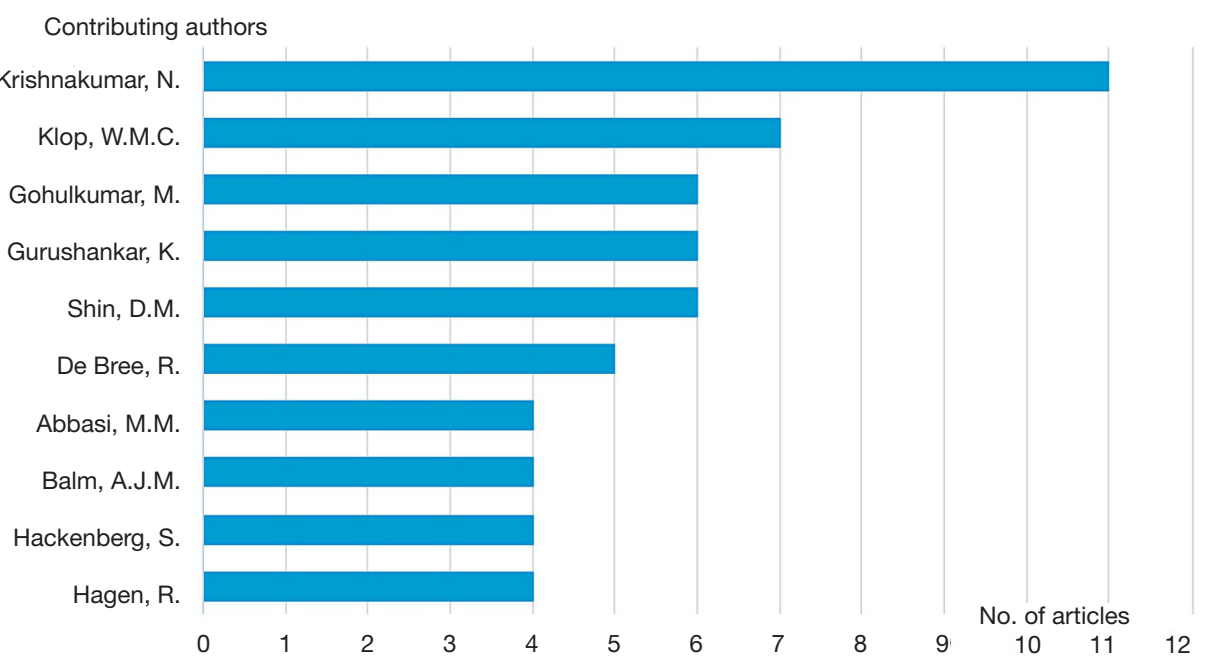

B

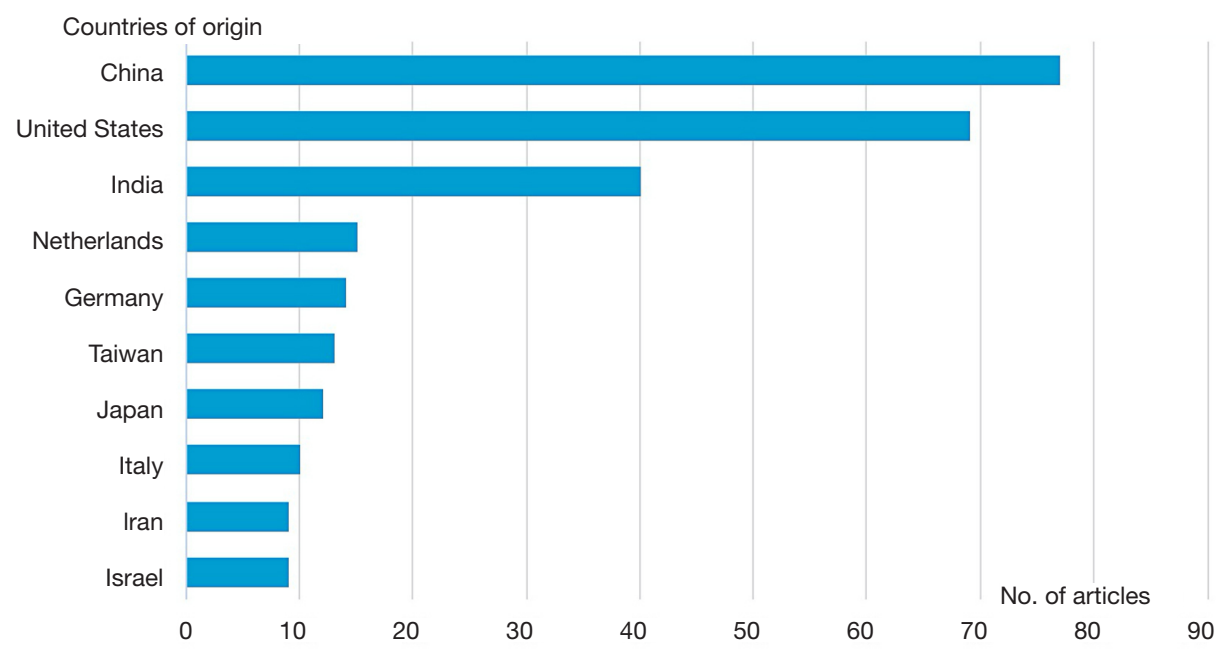

C

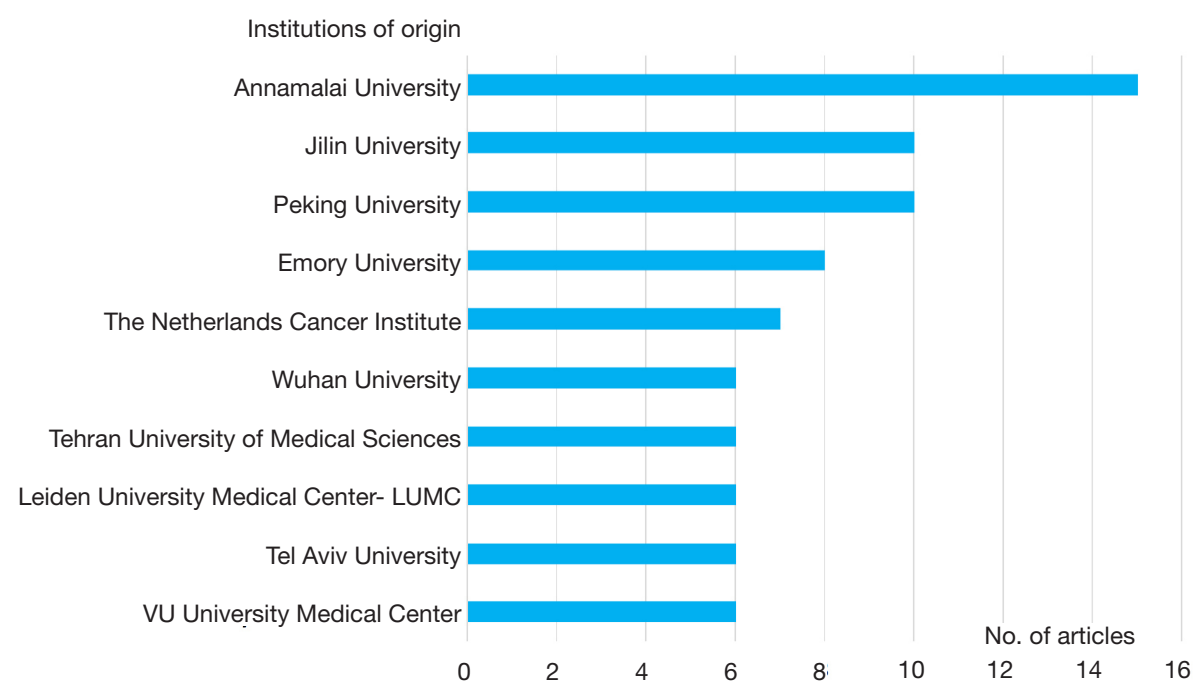

Figure 3 Information on the top 10 contributing authors (A), countries (B), and institutions (C) according to number of articles published (rank 1-10). 
A

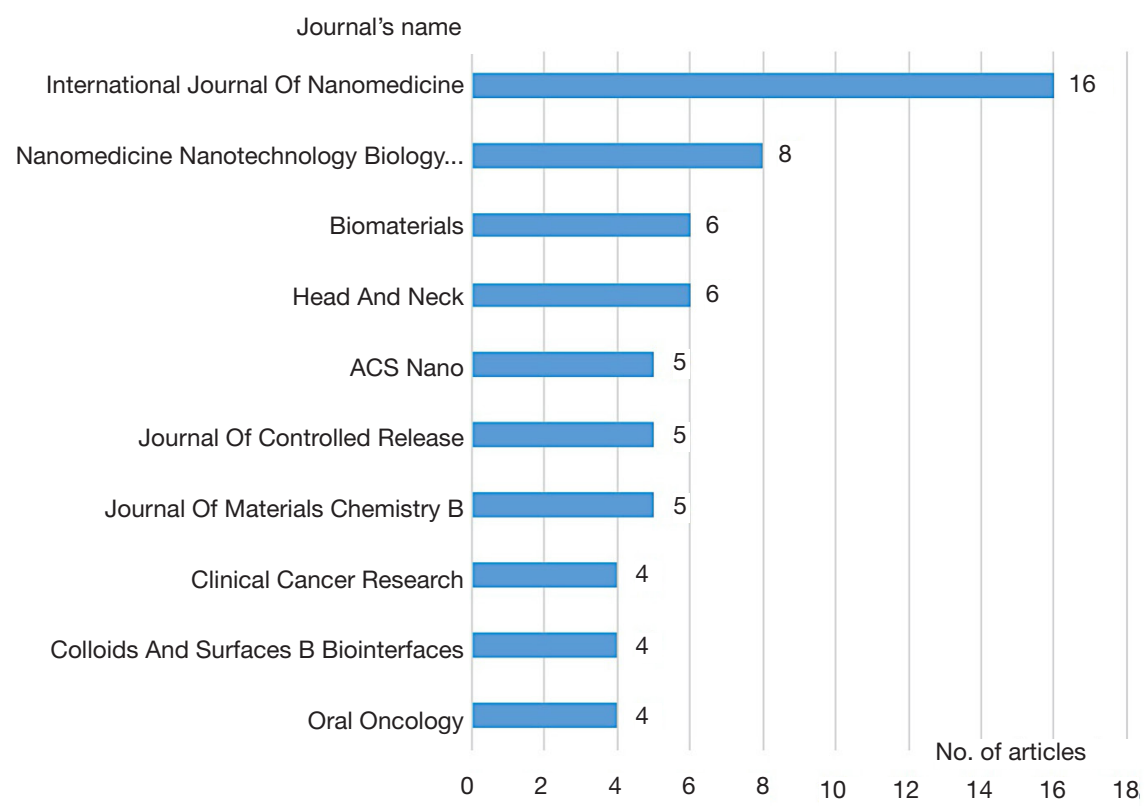

B

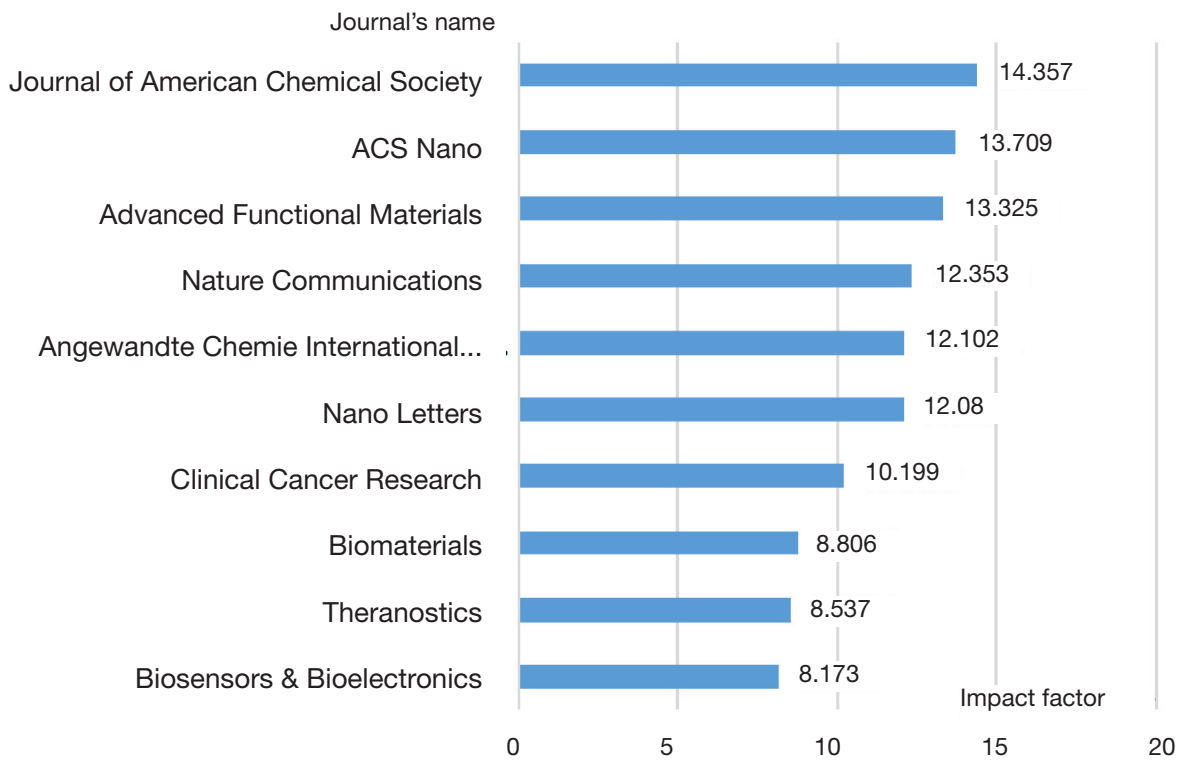

Figure 4 The top 10 journals according to number of articles published (A) and impact factors (IF) (B).

$(11,12)$. It is well known that both the IF of a journal and the citation rate of a paper are the main indicators for assessing their respective influence (20). Journals with a high IF are attractive to scholars when selecting a journal for publication of high-quality papers, while citation counts often indicate the extent a given study has been referred to by other scholars for use in their own studies (20). To the best of our knowledge, no bibliometric analysis has been performed regarding nanomedicine research in HNSCC. This is thus the first study to analyze the bibliometric characteristics and evaluate the citation trends of nanomedicine research in HNSCC, so as to provide the influence metrics in this field.

This study revealed insights into the main applications of nanomedicine research in HNSCC. Chemotherapy was the main application of nanotechnology in the therapy area 
of HNSCC, followed by chemoprevention, photodynamic therapy, photothermal therapy, and radiotherapy (Figure $2 A$ ). Conventional chemotherapy often results in multidrug resistance which limits its efficacy and exacerbates adverse drug-related side effects. Nanomedicine, which can provide enhanced drug efficiency and decreased toxicity, has emerged as an alternative to conventional therapeutics (5-7). Nanocarriers can improve the stability of drugs and targeted delivery, and offers the potential to reduce drug resistance via higher intracellular drug accumulation (7-9). Chemoprevention for HNSCC mainly uses natural products such as curcumin and ellagic acid, which have attracted significant attention in this field. Preclinical studies have revealed that some chemopreventive agents have promising anticancer properties (7). Nevertheless, natural compounds often have poor solubility and bioavailability, reducing their efficacy in clinical studies (7). To overcome this limitation, nanocarriers of chemopreventive compounds have been explored preclinically and have yielded some promising results (7). Applications of nanotechnology in HNSCC also offer the potential to improve the therapeutic efficacy and tolerability of photodynamic therapy, photothermal therapy, and radiotherapy $(21,22)$. However, only three phase I/II clinical studies on chemotherapy and one phase I clinical study on radiotherapy in HNSCC are available.

Sentinel node detection and non-invasive cancer diagnosis were the main applications of nanotechnology in the diagnostic area of HNSCC (Figure 2A). Nanocolloidal albumin-based positron emission tomography-computed tomography (PET-CT) lymphoscintigraphy for sentinel node detection in HNSCC, as an alternative to conventional imaging, was the main application of nanotechnology (22). Furthermore, an analysis of exhaled breath for diagnosing HNSCC using an array of nanomaterial-based sensors has also been explored (23). Additionally, an investigation of a biofunctionalized nanostructured zirconia-based biosensing platform for noninvasive oral cancer detection has been reported (24). Moreover, Raman spectroscopy, oral fluid-derived exosomes, saliva, and blood serum-based nanoparticles for noninvasive oral cancer detection have also been studied in the preclinical setting (15). However, no clinical studies are available that elucidate the diagnostic accuracy of nanocarriers in HNSCC in the clinical setting.

According to the journals with the largest number of articles in the field (rank 1-10), our information suggests that researchers often focus on nanotechnology-related journals to publish their studies. International fournal of
Nanomedicine and Biomaterials had the largest and third largest number of publications on nanomedicine research in HNSCC, respectively. Biomaterials and International Fournal of Nanomedicine also had the largest and second largest number of publications on nanomedicine research in TNBC (17). The top three contributing countries for nanomedicine research in HNSCC were China (28.2\%), the United States $(25.3 \%)$, and India (16.7\%), while the top three countries for nanomedicine research in TNBC were the United States (28.4\%), China (25.6\%), and India (13.3\%) (17). Consistently, the leading contributing institutions were from these three countries (Figure 3). These results can be attributed to the high level of financial support and the large number of researchers in these countries.

Although we attempted to minimize potential sources of bias, some limitations to this citation analysis may remain. First, we only used the Scopus database for analysis, and did not search other databases, partly because the Scopus database can automatically exclude self-citations. Furthermore, Scopus provides approximately 20\% more coverage compared to Web of Science, and provides more consistent and accurate results compared to Google Scholar $(25,26)$. Second, the number of citations does not directly represent the quality of an article, but facilitates a quantitative evaluation of the academic influence of an article in a targeted field. Researchers often tend to reference earlier highly cited articles independently of quality and content through a snowball effect. Third, the time of publication has a definite bias in the citation analysis, yet we calculated the citation count per year of each paper to clarify the paper's annual scientific impact.

\section{Conclusions}

The present study was the first to analyze the bibliometric characteristics of original articles on nanomedicine research in HNSCC. The results of this study not only provide a historical perspective on the scientific evolution within the field, but also revealed the trends and key topics of the field. We hope that the list of original articles in nanomedicine can provide a source of information for scholars and researchers. Overall, nanomedicine research in HNSCC is still in its infancy, and further investigations, especially clinical trials, are still required. Nevertheless, nanomedicine, as a cutting-edge technology is poised to create a paradigm shift in the early detection, prevention, 
and treatment of HNSCC.

\section{Acknowledgments}

The authors thank AME Editing Service for language editing.

Funding: This work was supported by National Natural Science Foundation of China (82074502), Science and Technology Commission of Shanghai Municipality (18411965300), Seed Fund (JYZZ073) of Shanghai Ninth People's Hospital, and Scientific Research Innovation Projects of Shanghai Jiao Tong University School of Medicine (201813010).

\section{Footnote}

Conflicts of Interest: All authors have completed the ICMJE uniform disclosure form (available at http://dx.doi. org/10.21037/tcr-20-2852). The authors have no conflicts of interest to declare.

Ethical Statement: The authors are accountable for all aspects of the work in ensuring that questions related to the accuracy or integrity of any part of the work are appropriately investigated and resolved.

Open Access Statement: This is an Open Access article distributed in accordance with the Creative Commons Attribution-NonCommercial-NoDerivs 4.0 International License (CC BY-NC-ND 4.0), which permits the noncommercial replication and distribution of the article with the strict proviso that no changes or edits are made and the original work is properly cited (including links to both the formal publication through the relevant DOI and the license). See: https://creativecommons.org/licenses/by-nc-nd/4.0/.

\section{References}

1. Bray F, Ferlay J, Soerjomataram I, et al. Global cancer statistics 2018: GLOBOCAN estimates of incidence and mortality worldwide for 36 cancers in 185 countries. CA Cancer J Clin 2018;68:394-424.

2. Huang SH, O'Sullivan B. Overview of the 8th Edition TNM Classification for Head and Neck Cancer. Curr Treat Options Oncol 2017;18:40.

3. Lala M, Chirovsky D, Cheng JD, et al. Clinical outcomes with therapies for previously treated recurrent/metastatic head-and-neck squamous cell carcinoma (R/M HNSCC):
A systematic literature review. Oral Oncol 2018;84:108-20.

4. Marur S, Forastiere AA. Head and Neck Squamous Cell Carcinoma: Update on Epidemiology, Diagnosis, and Treatment. Mayo Clin Proc 2016;91:386-96.

5. Calixto G, Bernegossi J, Fonseca-Santos B, et al. Nanotechnology-based drug delivery systems for treatment of oral cancer: a review. Int J Nanomedicine 2014;9: 3719-35.

6. Irimie AI, Sonea L, Jurj A, et al. Future trends and emerging issues for nanodelivery systems in oral and oropharyngeal cancer. Int J Nanomedicine 2017;12:4593-606.

7. Marcazzan S, Varoni EM, Blanco E, et al. Nanomedicine, an emerging therapeutic strategy for oral cancer therapy. Oral Oncol 2018;76:1-7.

8. Sah AK, Vyas A, Suresh PK, et al. Application of nanocarrier-based drug delivery system in treatment of oral cancer. Artif Cells Nanomed Biotechnol 2018;46:650-7.

9. Kakkar V, Verma MK, Saini K, et al. Nano drug delivery in treatment of oral cancer, a review of the literature. Curr Drug Targets 2019;20:1008-17.

10. Chen XJ, Zhang XQ, Liu Q, et al. Nanotechnology: a promising method for oral cancer detection and diagnosis. J Nanobiotechnology 2018;16:52.

11. Hachem LD, Mansouri A, Juraschka K, et al. Citation classics in neuro-oncology: assessment of historical trends and scientific progress. Neuro Oncol 2017;19:1158-72.

12. Liu W, Ma L, Song C, et al. Research trends and characteristics of oral lichen planus: a bibliometric study of the top-100 cited articles. Medicine (Baltimore) 2020;99:e18578.

13. Liu W, Zhang Y, Wu L, et al. Characteristics and trends of oral leukoplakia research: A bibliometric study of the 100 most cited articles. Medicine (Baltimore) 2019;98:e16293.

14. Pena-Cristóbal M, Diniz-Freitas M, Monteiro L, et al. The 100 most cited articles on oral cancer. J Oral Pathol Med 2018;47:333-44.

15. Foy JP, Bertolus C, Goudot P, et al. Bibliometric analysis of a century of research on oral erythroplakia and leukoplakia. J Oral Pathol Med 2018;47:388-95.

16. Liu W, Wu L, Zhang Y, et al. Bibliometric analysis of research trends and characteristics of oral potentially malignant disorders. Clin Oral Investig 2020;24:447-54.

17. Teles RHG, Moralles HF, Cominetti MR. Global trends in nanomedicine research on triple negative breast cancer: a bibliometric analysis. Int J Nanomedicine 2018;13:2321-36.

18. El-Sayed IH, Huang X, El-Sayed MA. Surface plasmon 
resonance scattering and absorption of anti-EGFR antibody conjugated gold nanoparticles in cancer diagnostics: applications in oral cancer. Nano Lett 2005;5:829-34.

19. Damascelli B, Cantù G, Mattavelli F, et al. Intraarterial chemotherapy with polyoxyethylated castor oil free paclitaxel, incorporated in albumin nanoparticles (ABI007): Phase I study of patients with squamous cell carcinoma of the head and neck and anal canal: preliminary evidence of clinical activity. Cancer 2001;92:2592-602.

20. Sobhy H. Social influence and peer review - impact factor and citation. EMBO Rep 2016;17:473.

21. Kim HS, Lee DY. Near-Infrared-Responsive Cancer Photothermal and Photodynamic Therapy Using Gold Nanoparticles. Polymers (Basel) 2018;10:961.

22. Huang DY, Hou YL, Yang SM, et al. Advances in

Cite this article as: Gao X, Wang S, Tian Z, Wu Y, Liu W. A bibliometric analysis on studies of nanomedicine applications in head and neck squamous cell carcinoma. Transl Cancer Res 2021;10(1):251-260. doi: 10.21037/tcr-20-2852 nanomedicine for head and neck cancer. Front Biosci (Landmark Ed) 2014;19:783-8.

23. Gruber M, Tisch U, Jeries R, et al. Analysis of exhaled breath for diagnosing head and neck squamous cell carcinoma: a feasibility study. Br J Cancer 2014;111:790-8.

24. Kumar S, Sharma JG, Maji S, et al. Nanostructured zirconia decorated reduced graphene oxide based efficient biosensing platform for non-invasive oral cancer detection. Biosens Bioelectron 2016;78:497-504.

25. Falagas ME, Pitsouni EI, Malietzis GA, et al. Comparison of PubMed, Scopus, Web of Science, and Google Scholar: strengths and weaknesses. FASEB J 2008;22:338-42.

26. Powell KR, Peterson SR. Coverage and quality: a comparison of Web of Science and Scopus databases for reporting faculty nursing publication metrics. Nurs Outlook 2017;65:572-8. 